\title{
Inhalt
}

Teil I: Rechtliche Grundlagen der Unfallbegutachtung

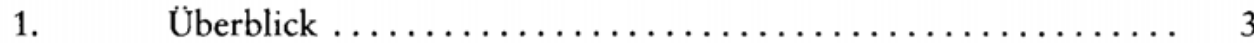

2. Gesetzliche Unfallversicherung $\ldots \ldots \ldots \ldots \ldots \ldots \ldots \ldots \ldots \ldots \ldots \ldots$

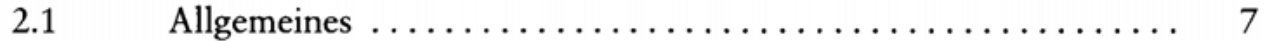

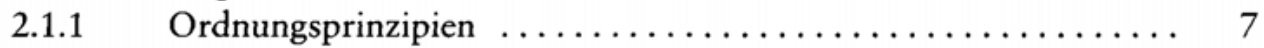

2.1.2 Berufsgenossenschaften und andere Versicherungsträger $\ldots \ldots \ldots \quad 10$

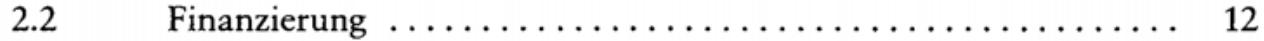

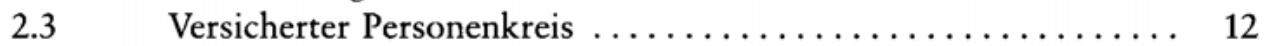

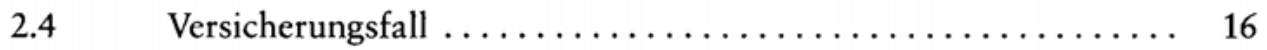

2.4.1 Arbeitsunfall .............................. 16

2.4.2 Ursachenzusammenhang und Beweisanforderungen ......... 17

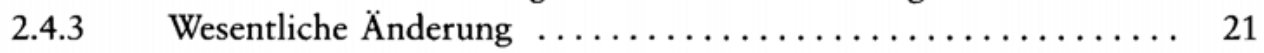

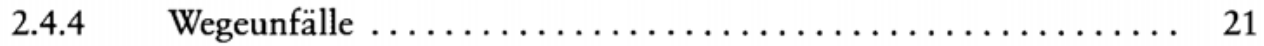

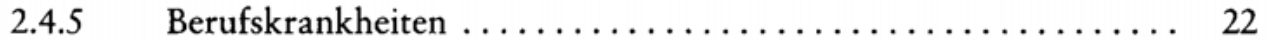

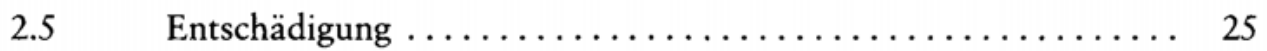

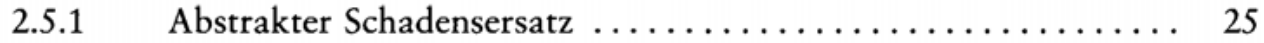

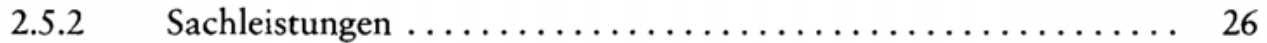

2.5.2.1 Heilbehandlung und medizinische Rehabilitation $\ldots \ldots \ldots \ldots 26$

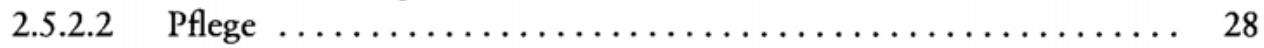

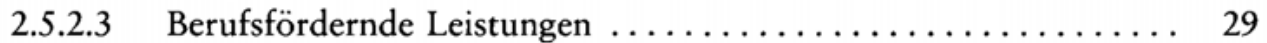

2.5.2.4 Soziale Rehabilitation ........................... 29

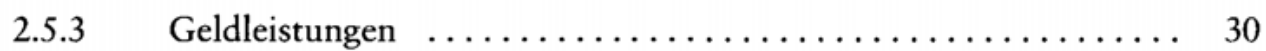

2.5.3.1 Verletztengeld - Übergangsgeld $\ldots \ldots \ldots \ldots \ldots \ldots \ldots \ldots \ldots \quad 30$

2.5.3.2 Rente .................................. 31

2.5.3.3 Vorläufige Rente - „Dauerrente“ ................. 34

2.5.3.4 Rente bei Kindern in Kindergärten, Schülern und Studierenden . 35

2.5.3.5 Leistungen an Hinterbliebene $\ldots \ldots \ldots \ldots \ldots \ldots \ldots \ldots . . \ldots \ldots$

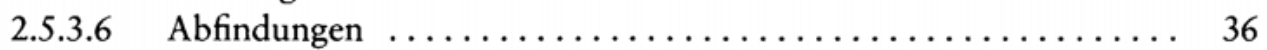

2.6 Verhältnis der Unfallversicherungsträger zu den Ärzten ....... 37

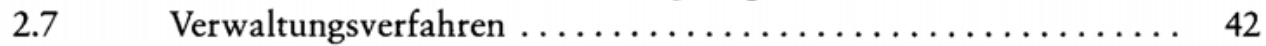

3. Gesetzliche Krankenversicherung $\ldots \ldots \ldots \ldots \ldots \ldots \ldots \ldots, 45$

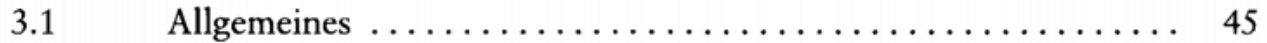

3.2 Träger der Krankenversicherung $\ldots \ldots \ldots \ldots \ldots \ldots \ldots \ldots, 46$

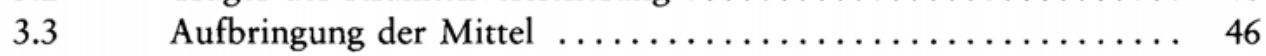

3.4 Versicherter Personenkreis ..................... 46

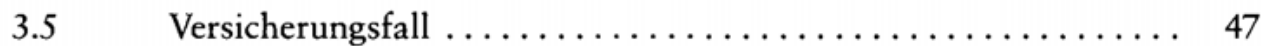


3.6 Leistungen der Krankenversicherung $\ldots \ldots \ldots \ldots \ldots \ldots \ldots, 48$

3.7 Beziehungen zur Unfallversicherung $\ldots \ldots \ldots \ldots \ldots \ldots \ldots \ldots, 48$

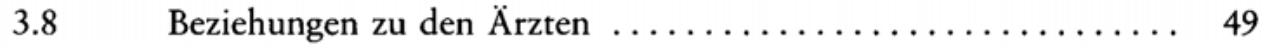

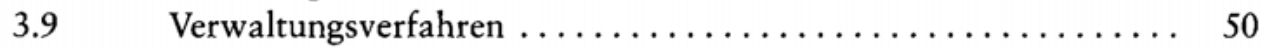

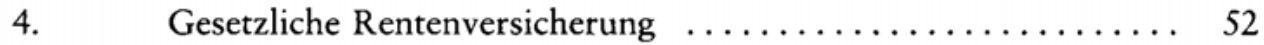

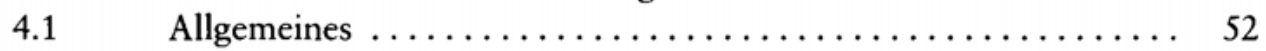

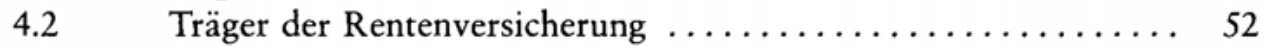

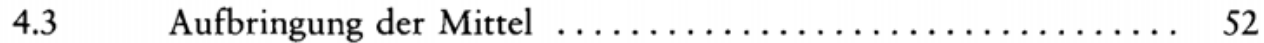

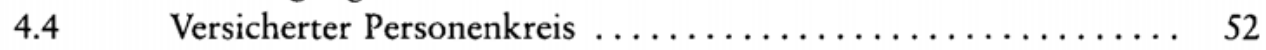

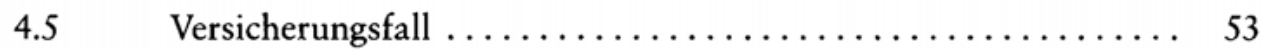

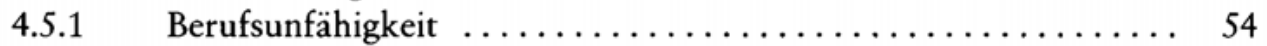

4.5.2 Erwerbsunfähigkeit ......................... 54

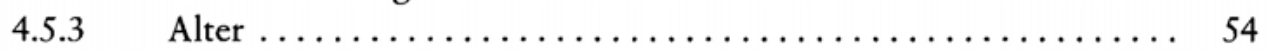

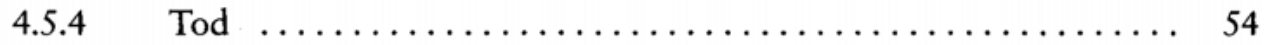

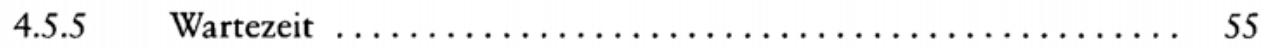

4.5.6 Besondere Versicherungsfälle der Knappschaftsversicherung .... 55

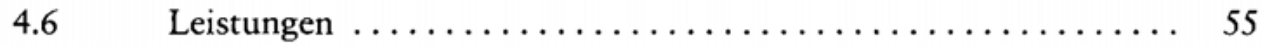

4.7 Beziehungen zur Unfallversicherung $\ldots \ldots \ldots \ldots \ldots \ldots \ldots \ldots \ldots$

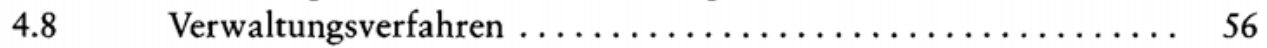

5. Soziales Entschädigungsrecht $\ldots \ldots \ldots \ldots \ldots \ldots \ldots \ldots \ldots \ldots$

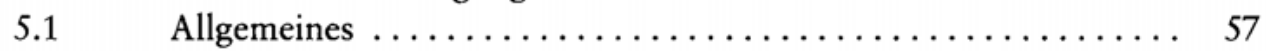

5.2 Soziale Entschädigung nach dem BVG ............. 57

5.3 Soziale Entschädigung nach anderen Gesetzen .......... 58

5.4 Umfang der Versorgungsleistungen bei Gesundheitsschäden ... 60

5.4.1 Heil- und Krankenbehandlung $\ldots \ldots \ldots \ldots \ldots \ldots \ldots \ldots, 60$

5.4 .2 Besondere Hilfen im Einzelfall $\ldots \ldots \ldots \ldots \ldots \ldots \ldots \ldots .61$

5.4.3 Renten und andere Geldleistungen $\ldots \ldots \ldots \ldots \ldots \ldots \ldots .62$

$5.5 \quad$ Aufbringung der Mittel zur Versorgung ............... 63

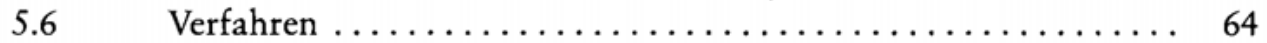

$5.7 \quad$ Hinweise für die Gutachter $\ldots \ldots \ldots \ldots \ldots \ldots \ldots \ldots \ldots \ldots$

6. Die Rehabilitation und ihre Träger $\ldots \ldots \ldots \ldots \ldots \ldots \ldots, 68$

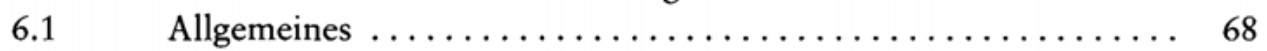

6.2 Träger der Rehabilitation $\ldots \ldots \ldots \ldots \ldots \ldots \ldots \ldots \ldots \ldots \ldots$

6.3 Maßnahmen und Leistungen zur Rehabilitation ............ 71

6.4 Zusammenwirken der Träger $\ldots \ldots \ldots \ldots \ldots \ldots \ldots \ldots, 72$

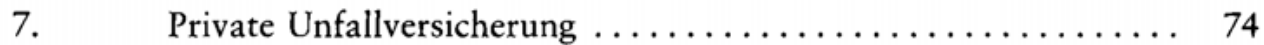

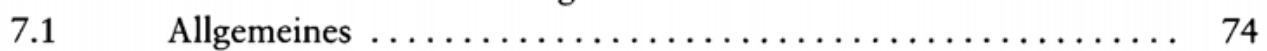

7.2 Träger der Versicherung - versicherter Personenkreis -

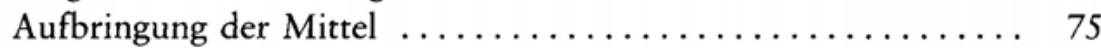




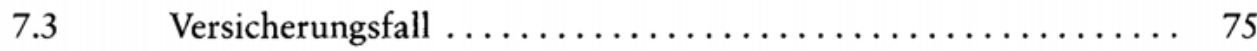

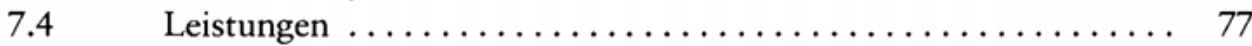

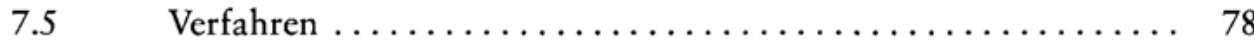

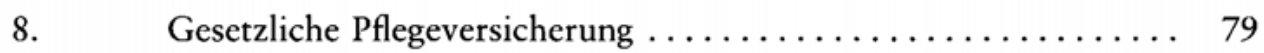

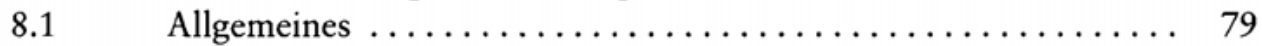

$8.2 \quad$ Versicherter Personenkreis ..................... 79

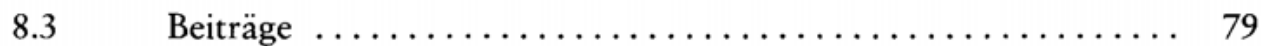

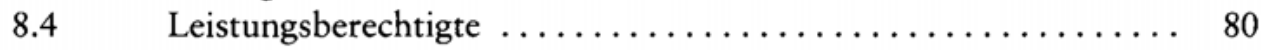

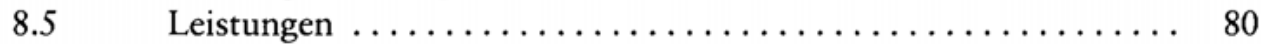

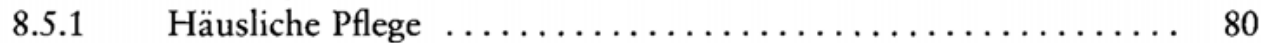

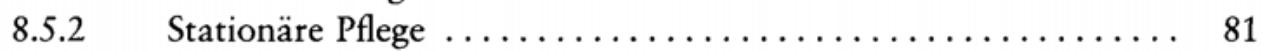

Teil II: Medizinische Gesichtspunkte der Unfallbegutachtung

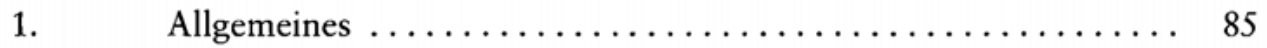

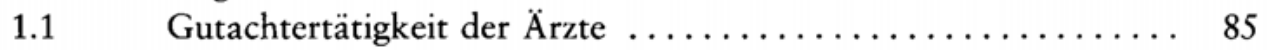

1.2 Rechtliche Stellung der Gutachter und ihre Aufgaben ....... 85

$1.3 \quad$ Formulierung der Gutachten $\ldots \ldots \ldots \ldots \ldots \ldots \ldots \ldots \ldots, 86$

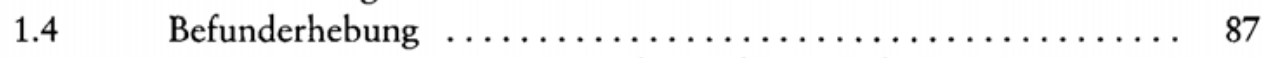

1.5 Entgegengesetzte Meinungen der Sachverständigen $\ldots \ldots \ldots .88$

1.6 Untersuchung der Verletzten für die Begutachtung .......... 89

1.7 Personenverwechslungen bei der Untersuchung ............ 89

$1.8 \quad$ Fehler und Irrtümer im Gutachten $\ldots \ldots \ldots \ldots \ldots \ldots \ldots \ldots .90$

$1.9 \quad$ Würdigung der Beweiskraft $\ldots \ldots \ldots \ldots \ldots \ldots \ldots \ldots \ldots \ldots$

$1.10 \quad$ Allgemeine Form des Gutachtens $\ldots \ldots \ldots \ldots \ldots \ldots \ldots . \quad 90$

1.11 Ausstellung von Bescheinigungen und Zeugnissen .......... 91

1.12 Auskunftspflicht der Ärzte $\ldots \ldots \ldots \ldots \ldots \ldots \ldots \ldots \ldots . \ldots . \ldots . \ldots 1$

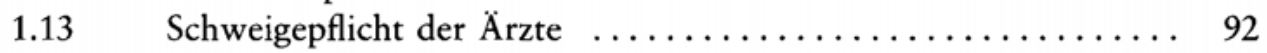

1.14 Sachkunde und Gutachternachwuchs $\ldots \ldots \ldots \ldots \ldots \ldots \ldots .93$

$1.15 \quad$ Schwierige Krankheitsfälle $\ldots \ldots \ldots \ldots \ldots \ldots \ldots \ldots \ldots . . \ldots 3$

1.16 Gebühren ................................... 94

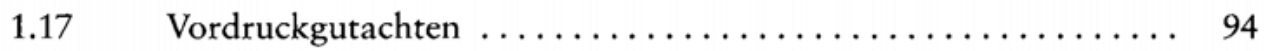

1.18 Form des freien Gutachtens .................... 95

1.19 Die Minderung der Erwerbsfähigkeit (MdE) ............. 99

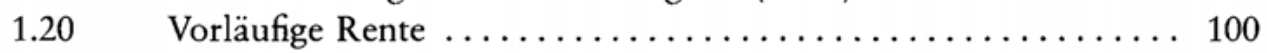

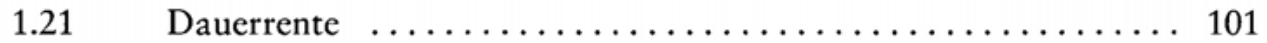

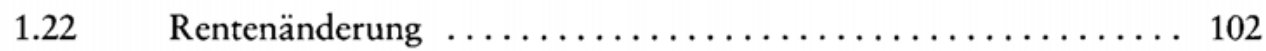

$1.23 \quad$ Vorschaden, Nachschaden $\ldots \ldots \ldots \ldots \ldots \ldots \ldots \ldots \ldots \ldots \ldots \ldots$

Wiederherstellende Behandlungsmaßnahmen -
Zumutbarkeit von Operationen ...................... 104 
1.25 Wichtige Untersuchungsmethoden für die Begutachtung ...... 105

1.26 Anleitung zur Benutzung der Meßblätter und Messung nach der Neutral-0-Methode ......................... 114

1.27 Simulation and Aggravation $\ldots \ldots \ldots \ldots \ldots \ldots \ldots \ldots \ldots \ldots \ldots \ldots$

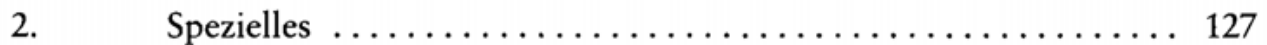

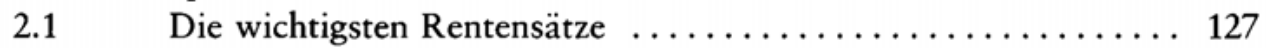

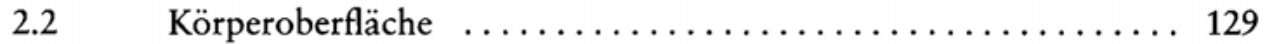

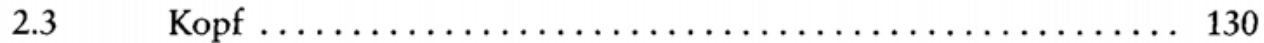

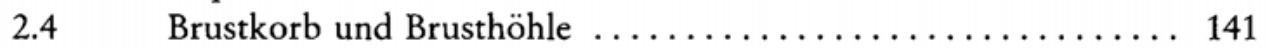

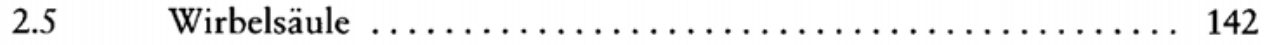

$2.6 \quad$ Becken .................................. 143

2.7 Bauchdecke und Bauchorgane .................... 143

$2.8 \quad$ Harnorgane ................................ 144

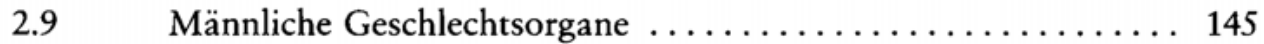

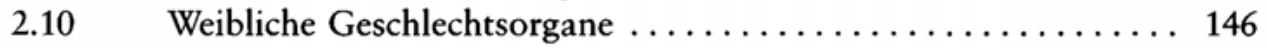

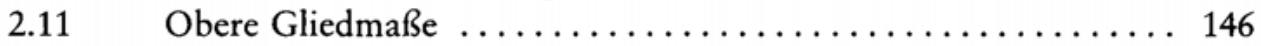

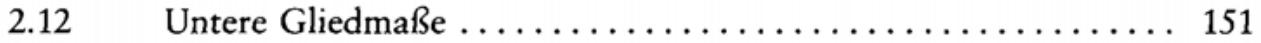

2.13 Anhaltspunkte für die Bemessung von Pflegegeld $\ldots \ldots \ldots \ldots .155$

3. Die Begutachtung von Fragen des ursächlichen Zusammenhanges zwischen Körperschäden und Arbeitsunfall ......... 157

3.1 Die Form der Gutachten $\ldots \ldots \ldots \ldots \ldots \ldots \ldots \ldots \ldots \ldots \ldots \ldots$

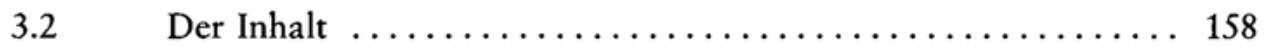

3.2.1 Rechtliche Voraussetzungen ...................... 158

3.2.2 Medizinische Voraussetzungen $\ldots \ldots \ldots \ldots \ldots \ldots \ldots \ldots \ldots \ldots$

3.3 Spezielles über die Begutachtung von Zusammenhangsfragen ... 161

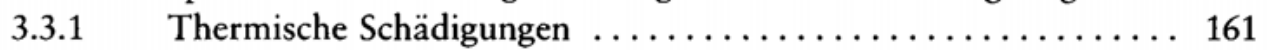

3.3.1.1 Erfrierungen und Verbrennungen $\ldots \ldots \ldots \ldots \ldots \ldots \ldots \ldots \ldots \ldots$

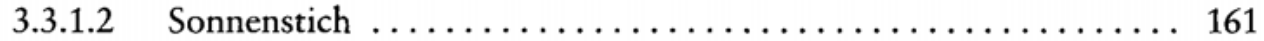

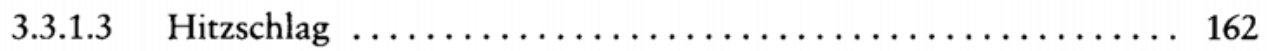

3.3.2 Verletzungen durch Einwirkungen des elektrischen Stroms .... 162

3.3.3 Akute Schädigungen durch Röntgenstrahlen, radioaktive Stoffe und andere ionisierende Strahlen $\ldots \ldots \ldots \ldots \ldots \ldots \ldots \ldots 2$

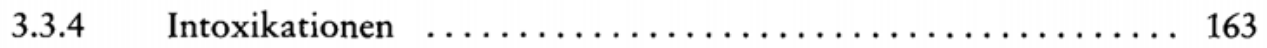

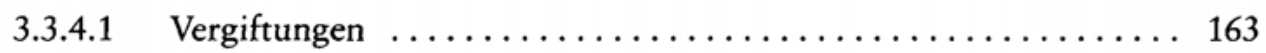

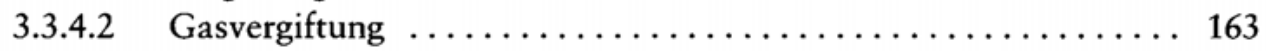

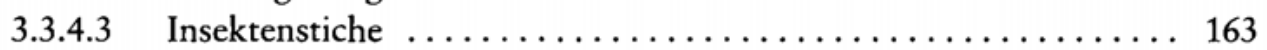

3.3.5 Infektionskrankheiten $\ldots \ldots \ldots \ldots \ldots \ldots \ldots \ldots \ldots \ldots \ldots \ldots \ldots \ldots \ldots$

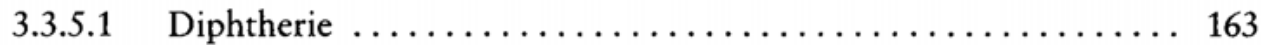

3.3.5.2 Typhus abdominalis, Paratyphus $\ldots \ldots \ldots \ldots \ldots \ldots \ldots \ldots \ldots \ldots$

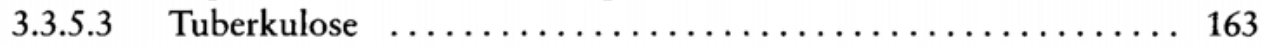

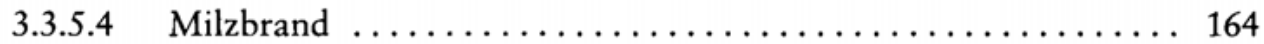




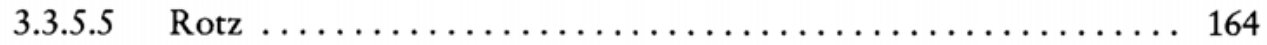

3.3.5.6 Aids, HIV-Infektion . . . . . . . . . . . . . . . . . . . . 164

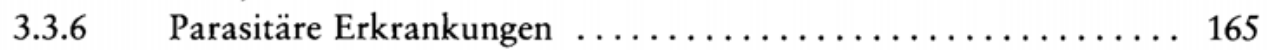

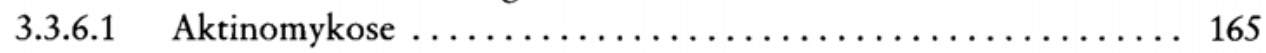

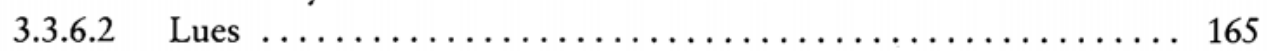

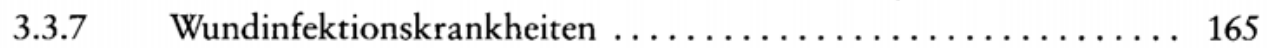

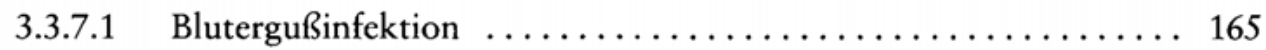

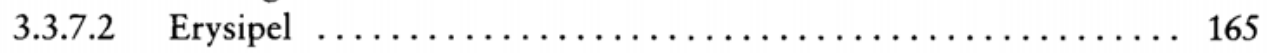

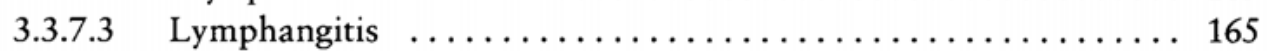

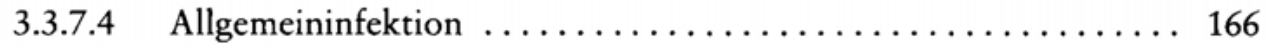

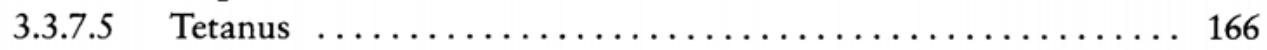

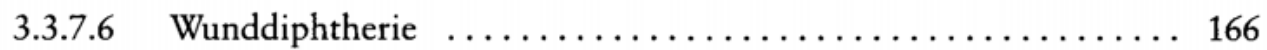

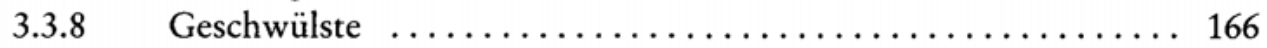

3.3.9 Stoffwechselkrankheiten und Krankheiten der endokrinen

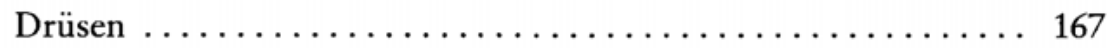

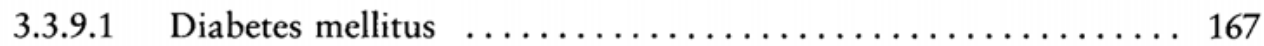

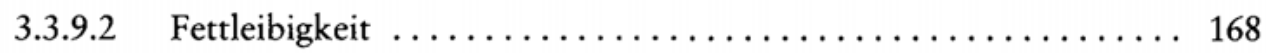

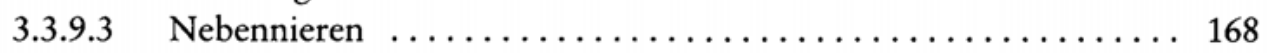

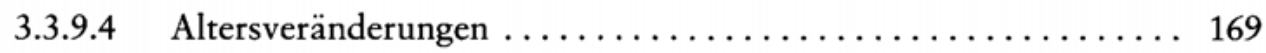

3.3.10 Erkrankungen des Blutes ......................... 169

3.3.10.1 Leukämie ................................... 169

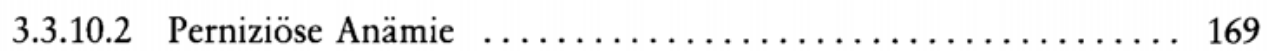

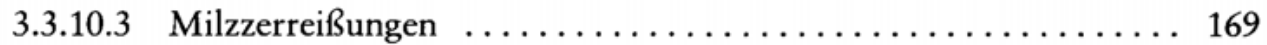

3.3.11 Erkrankungen des Gefäßsystems ..................... 169

3.3.11.1 Herzmuskel und Herzklappen . . . . . . . . . . . . . . . . 169

3.3.11.2 Aortenaneurysma . .......................... 170

3.3.11.3 Aneurysmen peripherer Gefäße $\ldots \ldots \ldots \ldots \ldots \ldots \ldots \ldots \ldots \ldots$

3.3.11.4 Krampfadern .............................. 171

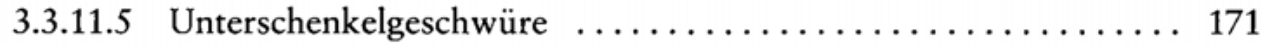

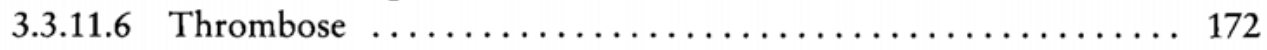

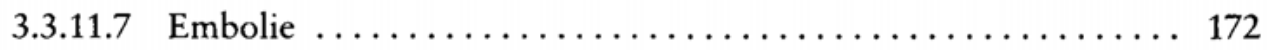

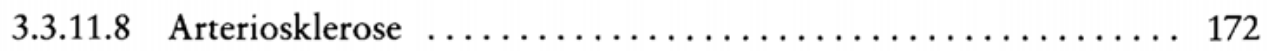

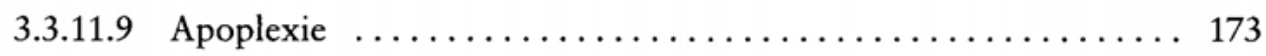

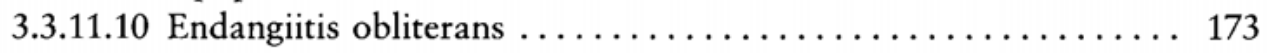

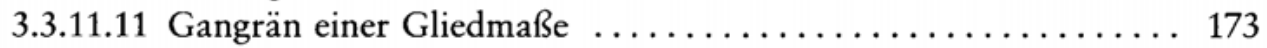

3.3.12 Erkrankungen der Atmungsorgane $\ldots \ldots \ldots \ldots \ldots \ldots \ldots \ldots \ldots$

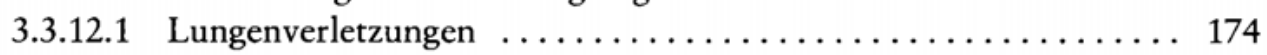

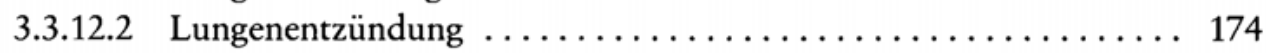

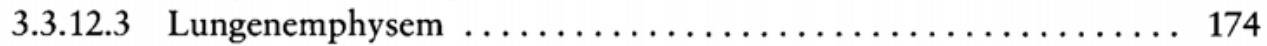

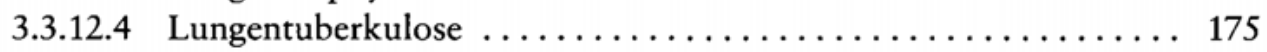

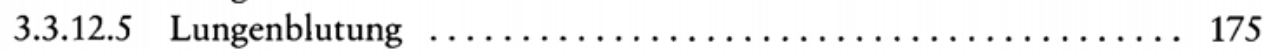

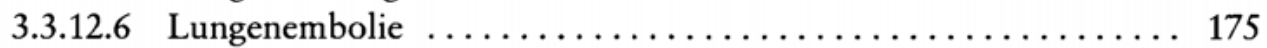

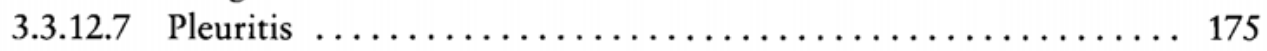




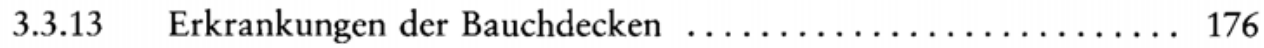

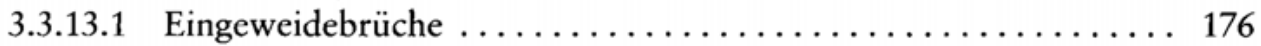

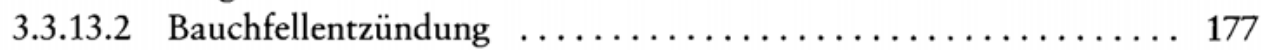

3.3.14 Erkrankungen des Magen- und Darmkanals ............ 177

3.3.14.1 Ösophagusdivertikel .......................... 177

3.3.14.2 Magen- und Zwölffingerdarmgeschwür ............... 177

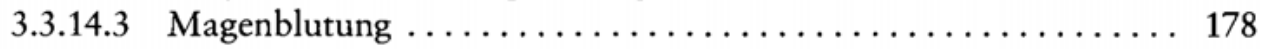

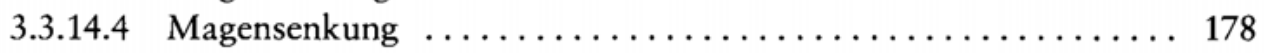

3.3.14.5 Magenkrebs .................................. 178

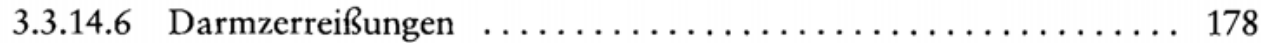

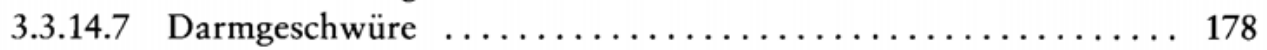

3.3.14.8 Darmverschluß ............................. 179

3.3.14.9 Appendizitis ................................ 179

3.3.14.10 Mastdarmvorfall ............................. 179

3.3.14.11 Mastdarmfisteln ............................. 179

3.3.15 $\begin{aligned} & \text { Erkrankungen der Leber, der Gallenwege und } \\ & \text { Bauchspeicheldrüse } \ldots \ldots \ldots \ldots \ldots \ldots \ldots \ldots \ldots \ldots\end{aligned}$

3.3.15.1 Virushepatitis .............................. 179

3.3.15.2 Chronische Hepatitis, Zirrhose .................... 179

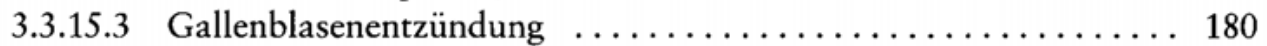

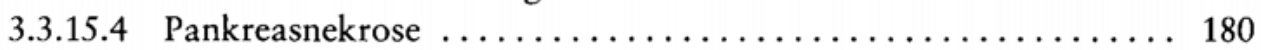

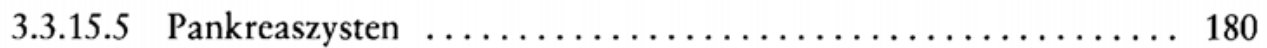

3.3.16 Erkrankungen des Harnsystems $\ldots \ldots \ldots \ldots \ldots \ldots \ldots \ldots \ldots \ldots$

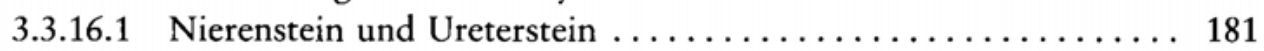

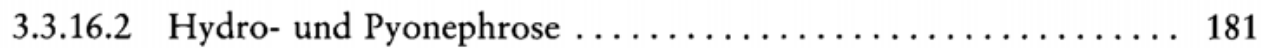

3.3.16.3 Neurogene Blasenentleerungsstörung $\ldots \ldots \ldots \ldots \ldots \ldots \ldots . \ldots \ldots 2$

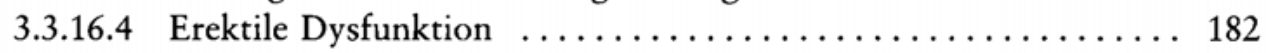

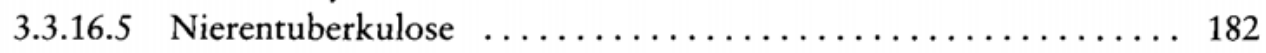

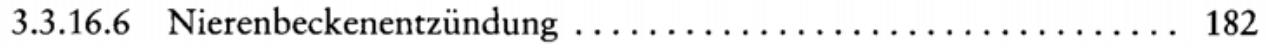

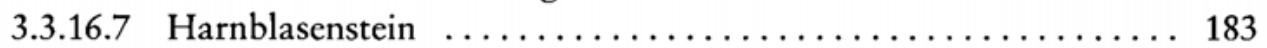

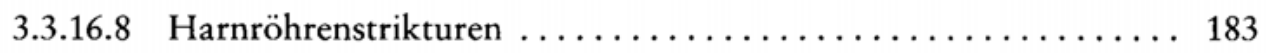

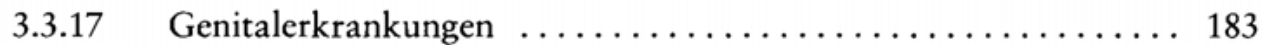

3.3.18 Erkrankungen der Haut und des Unterhautzellgewebes ....... 183

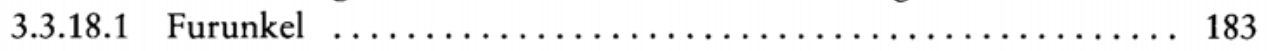

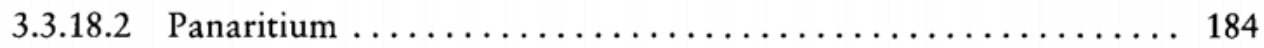

3.3.18.3 Zellgewebsentzündungen (Phlegmone) ............... 184

3.3.19 Erkrankungen der Muskeln, Sehnen und Schleimbeutel ....... 184

3.3.19.1 Muskelrisse .............................. 184

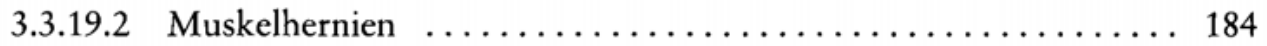

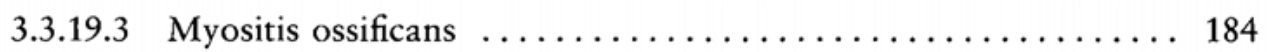

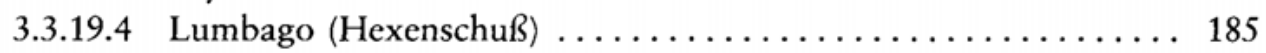

3.3.19.5 Bandscheibenvorfall im Bereich der Wirbelsäule ........... 185

3.3.19.6 Bizepssehnenriß ........................... 186 
3.3.19.7 Riß der Achillessehne ........................ 186

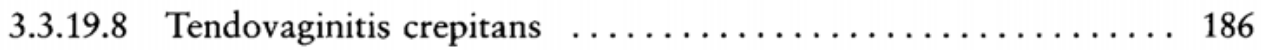

3.3.19.9 Dupuytrensche Kontraktur $\ldots \ldots \ldots \ldots \ldots \ldots \ldots \ldots \ldots \ldots \ldots \ldots \ldots$

3.3.19.10 Schleimbeutelentzündungen $\ldots \ldots \ldots \ldots \ldots \ldots \ldots \ldots \ldots \ldots \ldots \ldots$

3.3.19.11 Periarthritis humero-scapularis $\ldots \ldots \ldots \ldots \ldots \ldots \ldots \ldots \ldots \ldots$

3.3.20 Erkrankungen der Knochen und Gelenke $\ldots \ldots \ldots \ldots \ldots \ldots 188$

3.3.20.1 Akute Ostitis nach Weichteiltrauma .................. 188

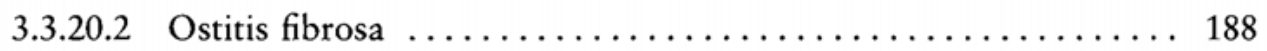

3.3.20.3 Tuberkulose der Knochen und Gelenke ............... 188

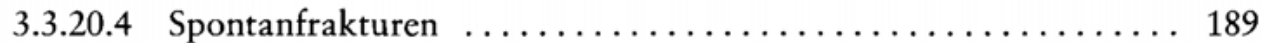

3.3.20.5 Dornfortsatzbruch (Schipperkrankheit) ................ 189

3.3.20.6 Navikularpseudarthrose der Hand ................... 189

3.3.20.7 Lunatumnekrose (Mondbeintod) $\ldots \ldots \ldots \ldots \ldots \ldots \ldots \ldots \ldots \ldots \ldots$

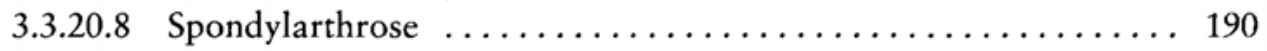

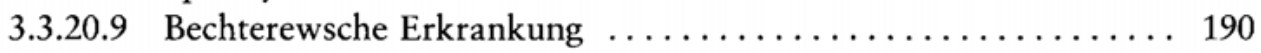

3.3.20.10 Spondylolisthesis (Wirbelgleiten) $\ldots \ldots \ldots \ldots \ldots \ldots \ldots \ldots \ldots \ldots$

3.3.20.11 Arthrosis deformans ......................... 190

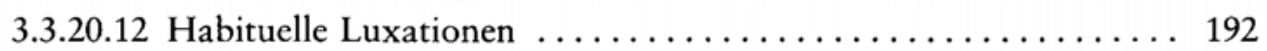

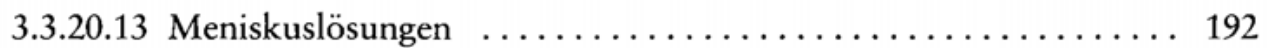

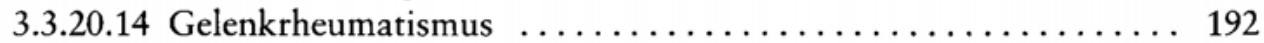

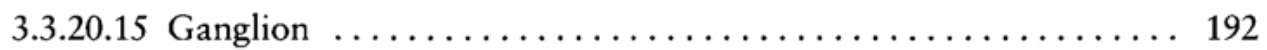

3.3.20.16 Gelenkmäuse ............................ 193

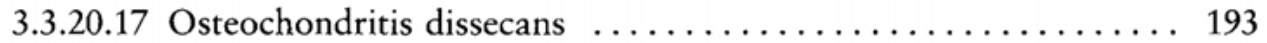

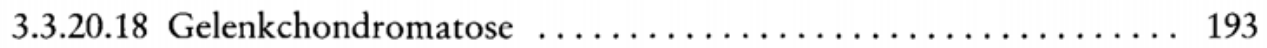

3.3.20.19 Gicht ................................... 193

3.3.20.20 Knochennekrosen ........................... 193

3.3.21 Erkrankungen des Nervensystems ..................... 194

3.3.21.1 Epilepsie .................................. 194

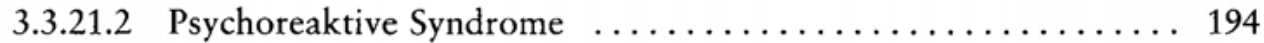

3.3.21.3 Hirnabszeß ................................ 194

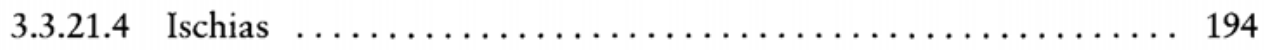

3.3.21.5 Hämatomyelie ............................. 195

3.3.21.6 Neurofibromatose ........................... 195

3.3.21.7 Progressive spinale Muskelatrophie $\ldots \ldots \ldots \ldots \ldots \ldots \ldots . \ldots 195$

3.3.22 Erkrankungen der Augen ....................... 195

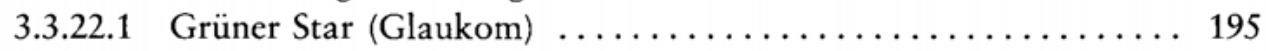

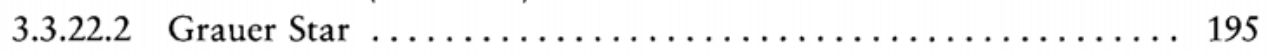

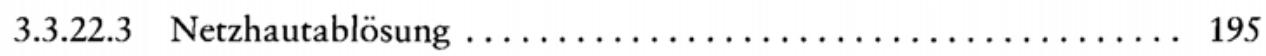


4. Die entschädigungspflichtigen Berufskrankheiten ......... 196

Nr. 1101 Erkrankungen durch Blei oder seine Verbindungen .... 196

Nr. 1102 Erkrankungen durch Quecksilber oder seine Verbindungen ....................... 197

Nr. 1103 Erkrankungen durch Chrom oder seine Verbindungen . 199

Nr. 1104 Erkrankungen durch Cadmium oder seine

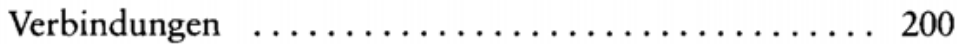

Nr. 1105 Erkrankungen durch Mangan oder seine Verbindungen 201

Nr.1106 Erkrankungen durch Thallium oder seine Verbindungen ........................ 201

Nr.1107 Erkrankungen durch Vanadium oder seine

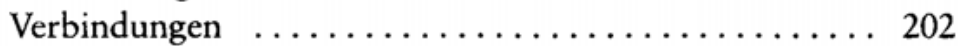

Nr. 1108 Erkrankungen durch Arsen oder seine Verbindungen .. 203

Nr. 1109 Erkrankungen durch Phosphor oder seine anorganischen Verbindungen ................ 204

Nr. 1110 Erkrankungen durch Beryllium oder seine

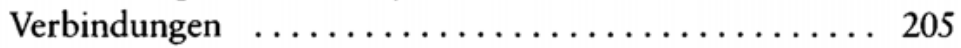

Nr. 1201 Erkrankungen durch Kohlenmonoxid ........... 206

Nr. 1202 Erkrankungen durch Schwefelwasserstoff ......... 208

Nr. 1301 Schleimhautveränderungen, Krebs oder andere Neubildungen der Harnwege durch aromatische Amine 208

Nr. 1302 Erkrankungen durch Halogenkohlenwasserstoffe .... 209

Nr. 1303 Erkrankungen durch Benzol, seine Homologe und Styrol ............................ 212

Nr. 1304 Erkrankungen durch Nitro- oder Aminoverbindungen des Benzols oder seiner Homologen oder ihrer Abkömmlinge ........................ 214

Nr. 1305 Erkrankungen durch Schwefelkohlenstoff ......... 215

Nr. 1306 Erkrankungen durch Methylalkohol (Methanol) ..... 216

Nr. 1307 Erkrankungen durch organische Phosphorverbindungen 217

Nr. 1308 Erkrankungen durch Fluor oder seine Verbindungen .. 218

Nr. 1309 Erkrankungen durch Salpetersäureester .......... 220

Nr. 1310 Erkrankungen durch halogenierte Alkyl-, Aryl- oder Alkylaryloxide ....................... 220

Nr.1311 Erkrankungen durch halogenierte Alkyl-, Aryl- oder Alkylarylsulfide ....................... 222

Nr. 1312 Erkrankungen der Zähne durch Säuren ......... 222

Nr. 1313 Hornhautschädigungen des Auges durch Benzochinon . 223

Nr. 1314 Erkrankungen durch para-tertiär-Butylphenol ...... 223

Nr. 1315 Erkrankungen durch Isocyanate, die zur Unterlassung aller Tätigkeiten gezwungen haben, die für die Entstehung, die Verschlimmerung oder das Wiederaufleben der Krankheit ursächlich waren oder sein können ... 225 
Nr.1316 Erkrankungen der Leber durch Dimethylformamid . . 227

Nr. 1317 Polyneuropathie oder Enzephalopathie durch organische Lösungsmittel oder deren Gemische ...... 228

Nr. 2101 Erkrankungen der Sehnenscheiden oder des Sehnengleitgewebes sowie der Sehnen- oder Muskelansätze, die zur Unterlassung aller Tätigkeiten gezwungen haben, die für die Entstehung, die Verschlimmerung oder das Wiederaufleben der Krankheit ursächlich waren oder sein können .......

Nr. 2102 Meniskusschäden nach mehrjährigen andauernden oder häufig wiederkehrenden, die Kniegelenke überdurchschnittlich belastenden Tätigkeiten

Nr. 2103 Erkrankungen durch Erschütterung bei Arbeit mit Druckluftwerkzeugen oder gleichartig wirkenden Werkzeugen oder Maschinen .............

Nr. 2104 Vibrationsbedingte Durchblutungsstörungen an den Händen, die zur Unterlassung aller Tätigkeiten gezwungen haben, die für die Entstehung, die Verschlimmerung oder das Wiederaufleben der Krankheit ursächlich waren oder sein können .......

Nr. 2105 Chronische Erkrankungen der Schleimbeutel durch

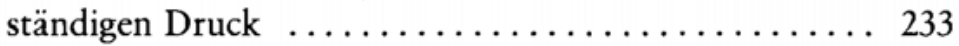

Nr. 2106 Drucklähmungen der Nerven . . . . . . . . . . . 234

Nr. 2107 Abrißbrüche der Wirbelfortsätze . . . . . . . . . . . . 234

Nr. 2108 Bandscheibenbedingte Erkrankungen der Lendenwirbelsäule durch langjähriges Heben oder Tragen schwerer Lasten oder durch langjährige Tätigkeit in extremer Rumpfbeugehaltung, die zur Unterlassung aller Tätigkeiten gezwungen haben, die für die Entstehung, die Verschlimmerung oder das Wiederaufleben der Krankheit ursächlich waren oder sein können .......

Nr. 2109 Bandscheibenbedingte Erkrankungen der Halswirbelsäule durch langjähriges Tragen schwerer Lasten auf der Schulter, die zur Unterlassung aller Tätigkeiten gezwungen haben, die für die Entstehung, die Verschlimmerung oder das Wiederaufleben der Krankheit ursächlich waren oder sein können .......

Nr. 2110 Bandscheibenbedingte Erkrankungen der Lendenwirbelsäule durch langjährige, vorwiegend vertikale Einwirkung von Ganzkörperschwingungen im Sitzen, die zur Unterlassung aller Tätigkeiten gezwungen haben, die für die Entstehung, die Verschlimmerung oder das Wiederaufleben der Krankheit ursächlich waren oder sein können 
Nr. 2111 Erhöhte Zahnabrasionen durch mehrjährige

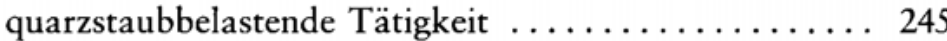

Nr. 2201 Erkrankungen durch Arbeit in Druckluft .......... 246

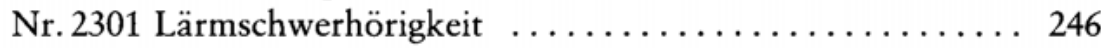

Nr. 2401 Grauer Star durch Wärmestrahlung ........... 248

Nr. 2402 Erkrankungen durch ionisierende Strahlen ... . . . . . . 249

Nr. 3101 Infektionskrankheiten, wenn der Versicherte im Gesundheitsdienst, in der Wohlfahrtspflege oder in einem Laboratorium tätig oder durch eine andere Tätigkeit der Infektionsgefahr in ähnlichem Maße besonders ausgesetzt war ............. 251

Nr.3102 Von Tieren auf Menschen übertragbare Krankheiten . . 254

Nr. 3103 Wurmkrankheit der Bergleute, verursacht durch Ankylostoma duodenale oder Strongyloides

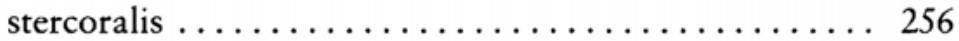

Nr. 3104 Tropenkrankheiten, Fleckfieber ............. 256

Nr. 4101 Quarzstaublungenerkrankung - Silikose . . . . . . . . 257

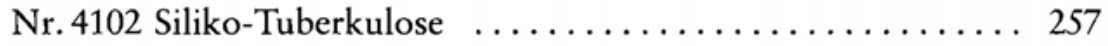

Nr.4103 Asbeststaublungenerkrankung (Asbestose) oder durch Asbeststaub verursachte Erkrankungen der

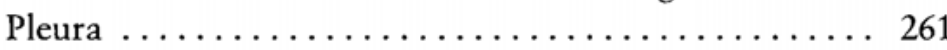

Nr. 4104 Lungenkrebs oder Kehlkopfkrebs ............. 263

- in Verbindung mit Asbeststaublungenerkrankung

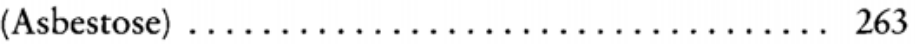

- in Verbindung mit durch Asbeststaub verursachte Erkrankung der Pleura ............... 263

- bei Nachweis der Einwirkung einer kumulativen Asbestfaserstaub-Dosis am Arbeitsplatz von mindestens 25 Faserjahren ................ 263

Nr. 4105 Durch Asbest verursachtes Mesotheliom des Rippenfells, des Bauchfells oder des Perikards ...... 266

Nr. 4106 Erkrankungen der tieferen Atemwege und der Lungen durch Aluminium oder seine Verbindungen ....... 267

Nr. 4107 Erkrankungen an Lungenfibrose durch Metallstäube bei der Herstellung oder Verarbeitung von Hartmetallen ............................ 268

Nr. 4108 Erkrankungen der tieferen Atemwege und der Lungen durch Thomasmehl (Thomasphosphat) ......... 269

Nr. 4109 Bösartige Neubildungen der Atemwege und der Lungen durch Nickel oder seine Verbindungen ..... 270

Nr. 4110 Bösartige Neubildungen der Atemwege und der Lungen durch Kokereirohgase . . . . . . . . . . . . 271 
Nr. 4111 Chronische obstruktive Bronchitis oder Emphysem von Bergleuten unter Tage im Steinkohlenbergbau bei Nachweis der Einwirkung einer kumulativen Dosis von in der Regel 100 Feinstaubjahren $\left[\left(\mathrm{mg} / \mathrm{m}^{3}\right) \times\right.$ Jahre $] \ldots 272$

Nr. 4201 Exogen-allergische Alveolitis . . . . . . . . . . . . 274

Nr. 4202 Erkrankungen der tieferen Atemwege und der Lungen durch Rohbaumwolle-, Rohflachs- oder Rohhanfstaub

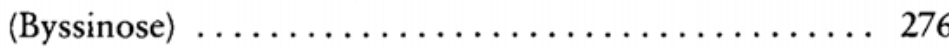

Nr. 4203 Adenokarzinome der Nasenhaupt- und Nasennebenhöhlen durch Stäube von Eichen- oder Buchenholz ........................ 277

Nr. 4301 Durch allergisierende Stoffe verursachte obstruktive Atemwegserkrankungen (einschließlich Rhinopathie), die zur Unterlassung aller Tätigkeiten gezwungen haben, die für die Entstehung, die Verschlimmerung oder das Wiederaufleben der Krankheit ursächlich waren oder sein können . . . . . . . . . . . . . . 277

Nr. 4302 Durch chemisch-irritativ oder toxisch wirkende Stoffe verursachte obstruktive Atemwegserkrankungen, die zur Unterlassung aller Tätigkeiten gezwungen haben, die für die Entstehung, die Verschlimmerung oder das Wiederaufleben der Krankheit ursächlich waren oder sein können

Nr. 5101 Schwere oder wiederholt rückfällige Hauterkrankungen, die zur Unterlassung aller Tätigkeiten gezwungen haben, die für die Entstehung, die Verschlimmerung oder das Wiederaufleben der Krankheit ursächlich waren oder sein können ...... 281

Nr. 5102 Hautkrebs oder zur Krebsbildung neigende Hautveränderungen durch Ruß, Rohparaffin, Teer, Anthrazen, Pech oder ähnliche Stoffe ......... 284

Nr. 6101 Augenzittern der Bergleute ................ 285

Anhang 1

Bildtafeln

\section{Anhang 2}

1 Die gesetzlichen Unfallversicherungsträger . . . . . . . . . . 305

2 Verzeichnis zur Ermittlung des zuständigen Unfallversicherungsträgers 319

3 Abkommen Ärzte/Unfallversicherungsträger (Ärzteabkommen), 1992 .. 325

4 Gemeinsame Richtlinien der Unfallversicherungsträger über Hilfsmittel

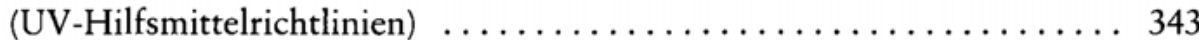


5 Gemeinsame Richtlinien der Spitzenverbände der Unfallversicherung

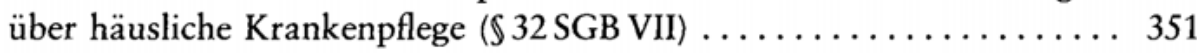

6 Gemeinsame Kraftfahrzeughilfe-Richtlinien der Verbände der

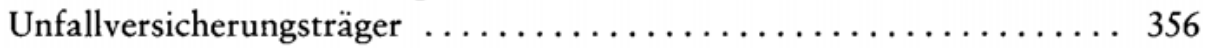

7 Gemeinsame Richtlinien der Unfallversicherungsträger über

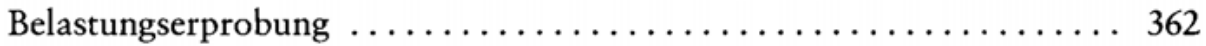

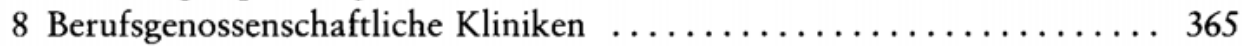

9 Grundsätze für die Bewilligung von Erholungsaufenthalten für Schwerstverletzte der Unfallversicherungsträger . . . . . . . . . . 367

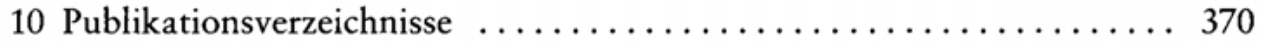

\section{Anhang 3}

Allgemeine Unfallversicherungs-Bedingungen (AUB 94), 1 T $88 \ldots \ldots \ldots .379$

Literatur 